\title{
Self-pumping Wavelength Conversion of Mixed Order PSK Signals by FWM to Realise Band Conversion
}

Kyle Bottrill, Francesca Parmigiani, David Richardson, Periklis Petropoulos

Optoelectronics Research Centre, University of Southampton, SO17 1BJ, UK

*krhb1g12@soton.ac.uk

\begin{abstract}
We present an FWM based wavelength conversion that differs from the typical approach in requiring no additional pump sources; two PSK signals are mixed in an HNLF, effectively pumping each other to facilitate wavelength conversion. The approach enables us to demonstrate band conversion as well as conversion of PSK signals of differing order.
\end{abstract}

\section{Introduction}

Wavelength conversion will be an essential function in the envisaged all-optical networks of the future and will be ubiquitous in wavelength division multiplexed (WDM) systems where it could be used to resolve channel contention and maximise bandwidth utilisation. Potential advantages offered by an all optical wavelength conversion scheme compared to the conventional electronic approach include high speed, low power consumption and format insensitivity.

Typically, four-wave mixing (FWM) schemes for wavelength conversion utilise a continuous wave $(\mathrm{CW})$ pump to translate a modulated signal to another wavelength. It is however, possible to mix two PSK signals such that each one pumps the other, resulting in a self-pumping FWM conversion scheme ${ }^{1}$. We present here just such a scheme which enables not only the simultaneous wavelength conversion of two QPSK signals, but also the possibility to perform 'band' conversion of two PSK signals - that is, two input signals may be shifted to longer or shorter wavelengths without their relative spacing being affected. Finally, we demonstrate the ability of this approach to perform wavelength conversion of PSK signals of differing orders.

\begin{tabular}{c|c|c|c}
$\phi_{+1}$ & $\phi_{+2}$ & $\phi_{+3}$ & $\phi_{+4}$ \\
\hline $2 \phi_{+0}-\phi_{-0}$ & $3 \phi_{+0}-2 \phi_{-0}$ & $4 \phi_{+0}-3 \phi_{-0}$ & $5 \phi_{+0}-4 \phi_{-0}$
\end{tabular}

\section{Background}

Figure 1 shows the result of mixing two high power quadrature phase shift keyed (QPSK) signals, $\phi_{-0}$ and $\phi_{+0}$, in a highly non-linear fibre (HNLF); a comb of QPSK signals is formed with an interval in frequency equal to the frequency difference between the original signals. Each signal has a time varying value of phase $\phi_{ \pm x}$, where the value of $x$ refers to the order of the FWM harmonic and the sign of $x$ denotes whether the harmonic is of longer wavelength $(+)$ or shorter wavelength $(-)$ than the original input signals.

For the first order FWM harmonics, it can be shown ${ }^{1,}$, that:

$$
\begin{aligned}
& \phi_{-1}=2 \phi_{-0}-\phi_{+0} \\
& \phi_{+1}=2 \phi_{+0}-\phi_{-0}
\end{aligned}
$$

These relationships can be applied recursively to generate phase relationships for the higher harmonics, of which the longer wavelength harmonics are listed in Table 1.

If $\phi_{-0}$ and $\phi_{+0}$ carry QPSK data, each with symbol alphabet $\{0, \pi / 2, \pi, 3 \pi / 2\}$, we can see from Table 1, that regardless of the value of $\phi_{+0}$, the value of $\phi_{+3}$ is always equal to $-3 \phi_{-0}$ (reducing to, $\phi_{-0}$ ). This is due to the coefficient of $\phi_{+0}$ collapsing the QPSK alphabet:

$4\{0, \pi / 2, \pi, 3 \pi / 2\} \rightarrow\{0,2 \pi, 4 \pi, 6 \pi\} \rightarrow\{0,0,0,0\}$. Effectively, the data stream carried by the signal $\phi_{-0}$ has been translated to the wavelength of $\phi_{+3}$.

The above method can be applied to the remaining harmonics listed in Table 1 , the results of which are shown by the lower,

Table 1: Phase relationships for red-shifted FWM harmonics.

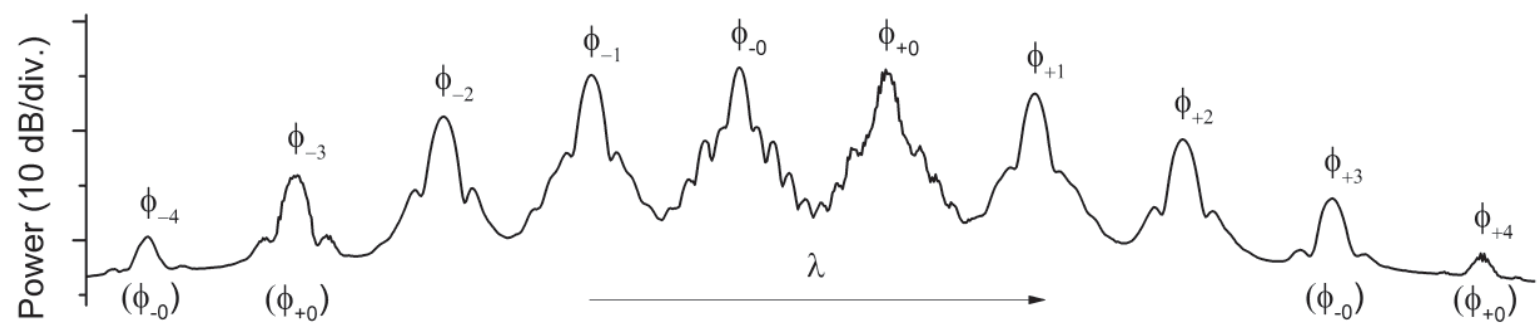

Figure 1: FWM comb for input of two QPSK signals $\left(\phi_{-0}\right.$ and $\left.\phi_{+0}\right)$ in HNLF. Upper labels denote harmonic nomenclature, lower labels in brackets 


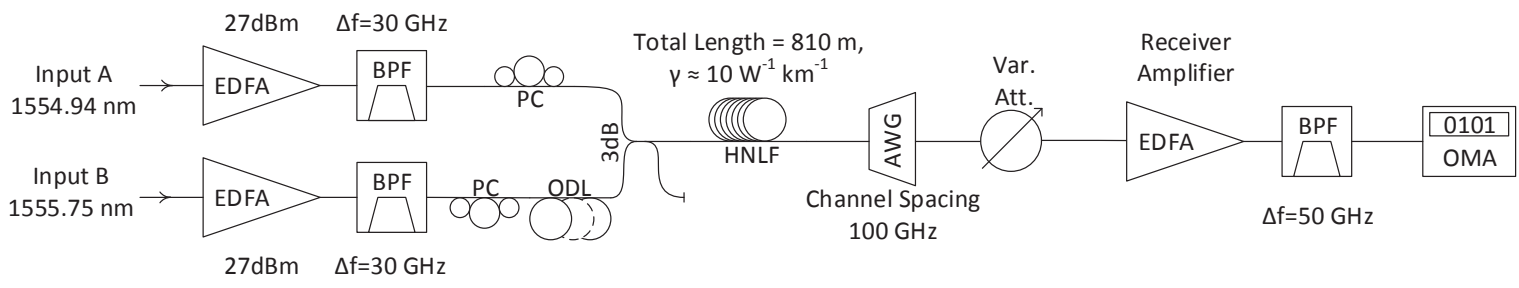

Figure 2: Experimental Setup

bracketed labels in Figure 1.

\section{Experimental Setup}

Figure 3 shows the experimental setup used in this investigation. The two PSK signals to be converted, of wavelength $1554.94 \mathrm{~nm}$ $(192.80 \mathrm{THz})$ and $1555.75 \mathrm{~nm}(192.70 \mathrm{THz})$, were launched into inputs $A$ and $B$ (as shown in the diagram). The details of the input signals are covered later in their relevant sections.

Firstly, the signals were amplified to high power using erbium doped fibre amplifiers (EDFAs). After amplification, band pass filters were used to reduce out-of-band noise and eliminate overlap of the two signals once they were coupled. This system resulted in the signals being launched into the HNLF with a power of about $14 \mathrm{dBm}$ each. The polarisation controllers were used to ensure that the two signals were copolarised in the HNLF in order to maximise the FWM efficiency. In addition, one of the input lines contained an optical delay line (ODL) so that the signals could be synchronised at their switching points to guarantee symbol overlap.

A low dispersion HNLF was used as the interaction medium to instigate four wave mixing. The total length of the HNLF came to $810 \mathrm{~m}$, and it had a nonlinear coefficient, $\gamma$, on the order of $10 \mathrm{~W}^{-1} \mathrm{~km}^{-1}$. The output of the HNLF (of which Figure 1 is an example) was then passed to an arrayed waveguide grating (AWG) to separate the channels (which, due to the $100 \mathrm{GHz}$ spacing of the input signals, also occur at intervals of $100 \mathrm{GHz}$ ).

The remainder of the setup forms the receiver. The optical modulation analyser (OMA) utilised a Kalman Filter algorithm ${ }^{3}$ in order to improve estimation of the carrier phase and polarisation of the received signal but otherwise no additional signal processing algorithms were applied to the detected signal.

\section{Results}

Wavelength Conversion with two QPSK Signals as Input

Firstly, we demonstrate simultaneous wavelength conversion of two QPSK signals. Two $10 \mathrm{GBaud} \mathrm{s}^{-1}$, NRZ-QPSK signals were

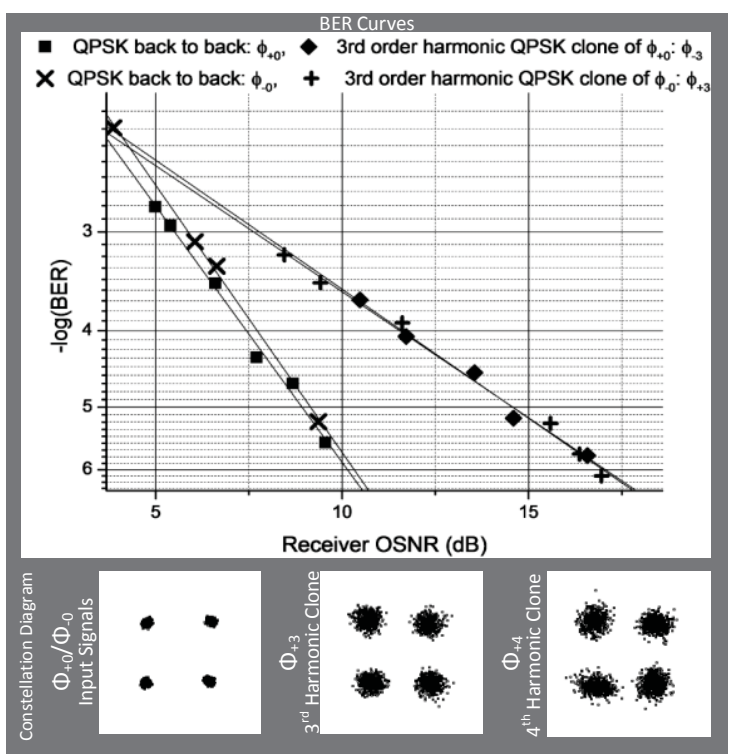

Figure 3: The result of using two QPSK signals as input. $\phi_{x}$ labels are with reference to Figure 1

used as inputs to the system. The signals were generated by modulating $\mathrm{CW}$ laser beams with the PRBS-31 data sequence with an IQ modulator. Figure 3 shows constellation plots and bit-error ratio (BER) curves for the two input signals $\left(\phi_{+0}\right.$ and $\left.\phi_{-0}\right)$ as well as the redshifted $3^{\text {rd }}$ harmonic $\left(\phi_{+3}\right)$ and red-shifted $4^{\text {th }}$ harmonic $\left(\phi_{+4}\right)$ QPSK clones.

The results for the blue-shifted signals are similar. The $4^{\text {th }}$ order harmonics exhibited an error floor resulting in a minimum BER of $1 \times 10^{-4}$ for an OSNR of $15 \mathrm{~dB}$ and, as such,

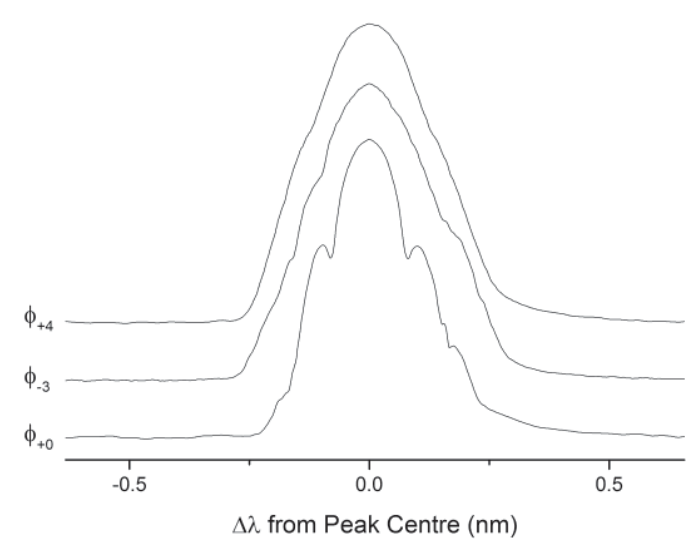

Figure 4: Spectra after receiver-side amplification and filtering for input QPSK signal as well as its 3rd and 4th order clones. Peaks have been normalised, centred and translated for better comparison. 
have been omitted from Figure 3 .

The FWM process converts the NRZ signal into a pulsed, pseudo-return-to-zero signal due to the signals' reduced intensity at symbol switching points combined with the intensity dependence of the FWM process, and this effectively shortens the symbol duration. This effect also manifests itself in the increased spectral width of signals that have undergone more four wave mixing events (Figure 4). Here, only the central lobes are visible in the spectra for the higher harmonics, as the other lobes are outside the bandwidth of the filter.

\section{Wavelength Conversion with one BPSK Signal and one QPSK Signal as Input}

To further investigate the potential of this scheme for band conversion of signals and to demonstrate the transparency of the approach to the size of the PSK alphabet of the signals, one of the input QPSK signals was replaced with a BPSK signal of identical symbol rate. The BPSK format is more robust to the wavelength conversion process and allowed for the recovery of the $4^{\text {th }}$ order QPSK signal with a BER of $<1 \times 10^{-7}$. Constellation plots and BER curves for the signals produced by the system are provided in Figure 1. The fourth order BPSK signal $\left(\phi_{-4}\right)$ shows a very good signal quality when compared to the $3^{\text {rd }}$ or $4^{\text {th }}$ order QPSK signals $\left(\phi_{+3}\right.$ or $\left.\phi_{+4}\right)$ in Figure 3.

From the BER curves, it can be seen that the performance of the wavelength conversion scheme with respect to BPSK signals is very good; the fourth order BPSK signal $\left(\phi_{-4}\right)$ suffers only a marginal power penalty. The QPSK signals suffer more upon conversion, however, the 3rd order harmonic $\left(\phi_{-3}\right)$ signal's power penalty of $\sim 0.5 \mathrm{~dB}$ is still quite respectable. Such a small power penalty for wavelength conversion highlights the potential of FWM for wavelength conversion.

\section{Conclusion}

We have demonstrated the wavelength conversion of two QPSK signals without additional pumps. Not only does this offer potential advantages in terms of energy efficiency as compared to other FWM wavelength conversion schemes, but it also eliminates the need for additional laser pump sources. In addition, the band conversion of two PSK signals was demonstrated. The

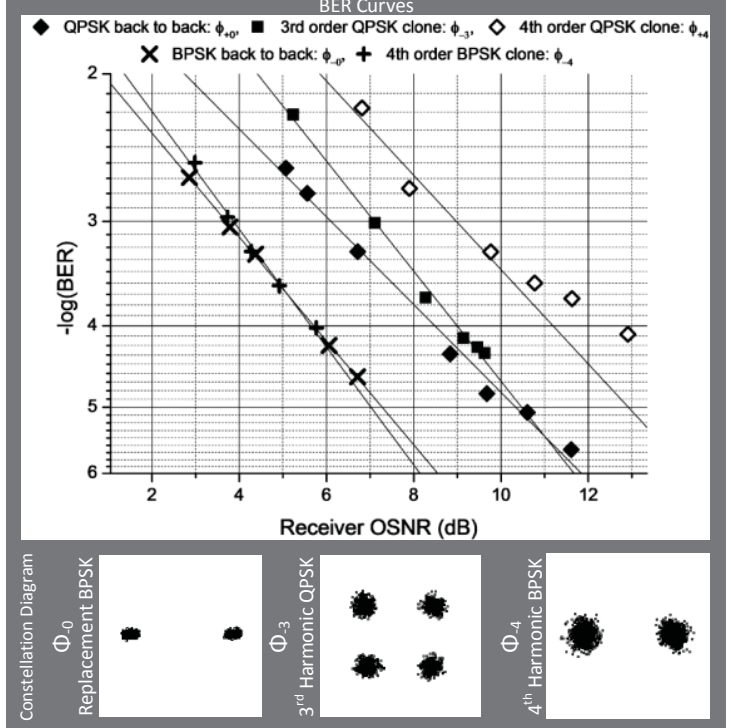

Figure 5: The result of using one BPSK signal and one QPSK signal as input. $\phi_{x}$ labels are with reference to Figure 1.

concept applied allows for the conversion of any two $M$-ary PSK signals, regardless of alphabet size, provided that the signals are of the same symbol rate.

During the mixing process, the signals are exposed to undesirable non-linear effects, such as self- and cross-phase modulation (SPM and XPM). In addition, as can also be seen from Figure 1, the non-linear processes result in the formation of a continuum, with cross-talk between neighbouring channels. These undesirable non-linear effects could be reduced, for instance, in systems that use a minimum shift keying format (MSK) ${ }^{4}$ which exhibits continuous phase, a near constant amplitude envelope and as a result, a narrow bandwidth for a given symbol rate.

The system may also benefit from higher HNLF input powers, which were restricted in our experiments due to technical limitations.

\section{References}

[1] Wu, Xue, et al. (2010), Conference on Lasers and Electro-Optics, OSA Technical Digest (CD), Optical Society of America, p. JThE57.

[2] Lu, Guo-Wei and Miyazaki, Tetsuya (2009), Optics Express, 17(16), pp. 13346-13353.

[3] B. Szafraniec, T. Marshall, B. Nebendahl, Technical Paper for Kalman Filter Based Modulation Analysis, Agilent Technologies, 2010 (online)

[4] Chen, Lemin et al. (2009), Optics Communications, 282(20), pp. 4045-4051. 